\title{
Chile frente a la regulación sobre medidas no arancelarias de la Organización Mundial del Comercio*
}

\author{
Chile and OMC's law on non tariff provisions
}

Sofía Boza ${ }^{* *}$ y Felipe Fernández ${ }^{* *}$

\section{Resumen}

En 1995, como uno de los resultados de la Ronda Uruguay de la Organización Mundial del Comercio, entra en vigor el Acuerdo sobre Obstáculos Técnicos al Comercio (OTC), cuyo objetivo principal es garantizar la potestad de los países de imponer este tipo de medidas, pero sin limitar el comercio más de lo necesario. Para ello, el Acuerdo establece una serie de principios que deben regir los OTC, así como mecanismos para promover una mayor transparencia. El objetivo principal de la presente investigación es exponer cuál ha sido la participación de Chile en estos mecanismos desde la entrada en vigor del Acuerdo hasta la actualidad. Palabras Clave. Organización Mundial del Comercio; Acuerdo OTC; mecanismos; participación; Chile.

\footnotetext{
Investigación financiada por el Programa U-INICIA Concurso de Reforzamiento de Inserción Productiva de Nuevos Académicos VID 2012 de la Universidad de Chile. Los autores agradecen los comentarios de los participantes en la Conferencia «Comercio Agrícola y América Latina: cuestiones, controversias y perspectivas» organizada por FLACSO (Argentina), el Instituto de Estudios Internacionales de la Universidad de Chile y el Centro de Derecho Económico Internacional del Instituto Tecnológico Autónomo de México (ITAM).

** Académica, Departamento de Economía Agraria, Facultad de Ciencias Agronómicas de la Universidad de Chile. <sofiaboza@u.uchile.cl>

*** Asistente de investigación del Proyecto U-Inicia «Evaluación de los efectos de las medidas sanitarias, fitosanitarias y técnicas en el comercio agroalimentario chileno». felferna@ ug.uchile.cl
}

Recibido el 23 de enero de 2014; aceptado el 10 de junio de 2014. 


\section{Abstract}

In 1995, as one of the results of the Uruguay Round of the World Trade Organization, the Agreement on Technical Barriers to Trade (TBT) came into force, whose main objective is to ensure that countries have the power to impose these measures, but without restricting trade more than necessary. The Agreement establishes a set of principles for the implementation of the TBT, as well as mechanisms to promote transparency. The main objective of this paper is to examine to what extent Chile has participated in these mechanisms from the entry into force of the Agreement to the present.

KEYwords. World Trade Organization; TBT Agreement; mechanisms; participation; Chile. 
Sofía Boza y Felipe Fernández • Chile frente a la regulación de las medidas...

\section{INTRODUCCIÓN}

Es medida no arancelaria cualquier medida, distinta de un arancel, que pueda afectar el comercio (Banco Mundial, 2008; UNCTAD, 2010). Según la Organización Mundial del Comercio (2012), las medidas no arancelarias incidirían principalmente en el precio, la cantidad o la calidad de los bienes transados. Dentro de esta última categoría destacan las medidas sanitarias y fitosanitarias (MSF) y los obstáculos técnicos al comercio (OTC), que exigen modificar las características técnicas de las importaciones. Con ellas se intenta mitigar distintos fallos del mercado, como las asimetrías de información entre el consumidor y el productor (Hobbs, 2010; Van Tongeren, Beghin \& Marette, 2009).

Con el fin de garantizar que los países puedan imponer este tipo de exigencias, pero siempre que no constituyan un medio de discriminación arbitrario o injustificable, o actúen como una restricción encubierta al comercio, en 1995 entraron en vigor el Acuerdo de Aplicación de Medidas Sanitarias y Fitosanitarias (Acuerdo MSF) y el Acuerdo sobre Obstáculos Técnicos al Comercio (Acuerdo OTC) de la Organización Mundial del Comercio. Ambos acuerdos continúan vigentes en la actualidad.

De manera precisa, el Acuerdo OTC tiene como objetivo garantizar que los países miembros puedan aplicar a las importaciones los requisito que sean necesarios para proteger la seguridad nacional, prevenir prácticas engañosas, proteger la salud o seguridad humanas, la vida o la salud animal o vegetal y el medio ambiente. No obstante, todo ello siempre que, como se dijo, no se generen obstáculos innecesarios al comercio internacional.

Para viabilizar lo anterior, el Acuerdo OTC establece una serie de principios que deben tener presentes las autoridades nacionales dotadas de capacidad regulatoria en la materia. Asimismo, para promover la transparencia y la construcción de consenso se establecen los procedimientos que deben seguirse para informar sobre la intención de un país de aplicar una medida determinada y se crea un Comité sobre Obstáculos Técnicos al Comercio (Comité OTC) que deberá recoger en sus reuniones las preocupaciones de los países miembros y las especificidades de las controversias ante el Órgano de Solución Diferencias de la OMC.

Chile, como país signatario del Acuerdo OTC, ha sido (y es) partícipe del proceso señalado. En este contexto, dada la importancia que revisten los ingresos derivados de las exportaciones para la economía chilena, así como su focalización en mercados de alta exigencia, resulta de especial interés examinar cuál ha sido el papel del país como miembro del Acuerdo. Para ello en el presente trabajo se realizará en primer lugar una revisión detallada del Acuerdo OTC, considerando su origen, ámbito, principios e institucionalidad, para a continuación exponer cuál ha sido la participación de Chile durante el 
periodo 1995-2012 en los mecanismos adoptados para el caso de los OTC.

\section{El ACUERdo SObre Obstáculos Técnicos Al Comercio}

La preocupación por la calidad e inocuidad de los productos importados ya se encontraba presente en el Acuerdo General sobre Aranceles Aduaneros y Comercio (GATT) de 1947, que en su artículo XX establece la facultad de los países de adoptar las medidas que se considere necesarias para proteger la salud y la vida humana y animal, así como la preservación de la vida vegetal. Sin embargo, solo en la VII Conferencia del GATT, denominada Ronda de Tokio debido a que se inauguró en 1973 en dicha ciudad, se suscribió un acuerdo específico al respecto. El Acuerdo sobre Obstáculos Técnicos al Comercio, también conocido como "Código de Normas», fue suscrito por treinta y dos países (partes contratantes del GATT) de los más de noventa presentes en la Ronda.

El Acuerdo OTC de la Ronda de Tokio fue pionero en el establecimiento de un marco de actuación para preparar, adoptar y aplicar reglamentos técnicos y normas, así como en la creación de procesos de evaluación de conformidad y certificación relacionados no solo con la protección de la salud humana, animal y vegetal, sino también con la conservación del medio ambiente y la prevención de prácticas engañosas. Con el fin de que estas medidas no provoquen restricciones innecesarias al comercio, el Acuerdo promueve, entre otras cosas, el respeto a los principios de transparencia y armonización. El primero de ellos se expresa en la obligación de los países signatarios de informar sobre su intención de introducir nuevas medidas o realizar cambios sustanciales en las existentes, en tiempo suficiente para poder considerar las observaciones que formulen las demás partes. Respecto del principio de armonización, señala la primacía del uso de las normas internacionales como base para el diseño de regulaciones técnicas y normas a nivel nacional, salvo el caso que justificadamente se consideren inapropiadas.

Pese a que, como se dijo, el Acuerdo OTC de la Ronda de Tokio constituyó un importante hito en la definición de un marco normativo relativo a los requisitos técnicos en el comercio, una de sus principales limitantes fue su baja adhesión. Además, el Acuerdo no se refería a ningún sector específico. En este contexto, la Declaración de Punta del Este de 1986, que dio inicio a la Ronda Uruguay, establece como tema de negociación, dentro del comercio agrícola, la reducción del impacto negativo de las normas y barreras sanitarias y fitosanitarias. Con este fin, en 1988 se constituyó el Grupo de Trabajo de las Reglamentaciones Sanitarias y Fitosanitarias, que al cabo de dos años había generado un primer borrador de acuerdo. Dado que varios puntos importantes habían quedado fuera de este borrador inicial, en 1991 Arthur Dunkel, Director General del GATT, presentó el conocido como «Tex- 
Sofía Boza y Felipe Fernández • Chile frente a la regulación de las medidas...

to Dunkel» (FAO, 2000), que sirvió de base al Acuerdo sobre Aplicación de Medidas Sanitarias y Fitosanitarias (Acuerdo MSF), aprobado en la sesión final de la Ronda Uruguay, en 1994, celebrada en la ciudad de Marrakech, Marruecos. En la misma oportunidad se suscribió el nuevo Acuerdo sobre Obstáculos Técnicos al Comercio. Prácticamente la totalidad de los 123 países que participaron en la Ronda Uruguay aprobaron sus resultados finales y, por tanto, los Acuerdos MSF y OTC, situación muy distinta de la que se había dado con el Acuerdo OTC. en 1979. Además, con la entrada en vigor del Acuerdo MSF las medidas en inocuidad alimentaria, protección de la salud animal $\mathrm{y}$ del patrimonio fitosanitario quedaran prioritariamente bajo él.

Por su parte, según se establece en su Preámbulo, el nuevo Acuerdo OTC centra la atención en garantizar que los países puedan adoptar las medidas necesarias para asegurar, además de los aspectos señalados en el Acuerdo
MSF, la calidad de las exportaciones, la protección del medio ambiente y la prevención de prácticas que puedan inducir a error, añadiendo el artículo 2.2 del Acuerdo a estos objetivos la protección de la seguridad nacional. Al igual que en el Acuerdo OTC de 1979, las medidas a que se refiere el nuevo acuerdo son tanto los reglamentos técnicos como las normas y procedimientos de evaluación de conformidad. La principal diferencia entre los dos primeros reside en su obligatoriedad: mientras que los reglamentos técnicos son de debido cumplimiento, las normas son de carácter voluntario. Sin embargo, ambos establecen las características de los productos, procesos o métodos de producción, así como requerimientos referentes a terminología, símbolos, embalaje, marcado o etiquetado. Por su parte, los procedimientos de evaluación de conformidad se refieren a los métodos para verificar que las importaciones cumplen con los reglamentos técnicos y/o las normas.

Cuadro 1. Tipos de medidas en el ámbito del Acuerdo OTC

\begin{tabular}{|l|l|}
\hline Tipo de medida & Definición según el Acuerdo OTC \\
\hline $\begin{array}{l}\text { Reglamento } \\
\text { técnico }\end{array}$ & $\begin{array}{l}\text { «Documento en el que se establecen las características de un } \\
\text { producto o los procesos y métodos de producción con ellas } \\
\text { relacionados, con inclusión de las disposiciones administrativas } \\
\text { aplicables, y cuya observancia es obligatoria» (Acuerdo OTC, } \\
\text { Anexo 1) }\end{array}$ \\
\hline Norma & $\begin{array}{l}\text { «Documento aprobado por una institución reconocida, que prevé, } \\
\text { para un uso común y repetido, reglas, directrices o características } \\
\text { para los productos o los procesos y métodos de producción } \\
\text { conexos, y cuya observancia no es obligatoria» (Acuerdo OTC, } \\
\text { Anexo 2) }\end{array}$ \\
\hline $\begin{array}{l}\text { Procedimiento } \\
\text { de evaluación de } \\
\text { conformidad }\end{array}$ & $\begin{array}{l}\text { «Todo procedimiento utilizado, directa o indirectamente, para } \\
\text { determinar que se cumplen las prescripciones pertinentes de los } \\
\text { reglamentos técnicos o normas» (Acuerdo OTC, Anexo 3) }\end{array}$ \\
\hline
\end{tabular}


Paralelamente con lo señalado en el párrafo anterior, el Acuerdo OTC establece que las medidas implementadas por los países miembros no deben constituir un medio de discriminación arbitrario o injustificado entre países de similares condiciones, ni una restricción encubierta al comercio internacional. Para evitar lo anterior, además de la transparencia y la armonización se establecen varios principios rectores como la no discriminación, la prevención de obstáculos innecesarios, la equivalencia y reconocimiento mutuo y la asistencia técnica y trato diferenciado a los miembros en desarrollo.

A continuación se detalla cada uno de los principios mencionados.

\section{a) No discriminación}

$\mathrm{La}$ «no discriminación» en el Acuerdo OTC responde a los principios de Nación más favorecida y trato nacional previstos desde el GATT 1947 (artícu$\operatorname{los} 1$ y 3$)$.

De manera específica, el Acuerdo OTC establece que, en materia de medidas técnicas, los productos importados deben ser objeto de un trato equivalente al otorgado a productos similares ${ }^{1}$ de

1 El Acuerdo OTC no establece de manera explícita a qué se refiere con "productos similares", no obstante de la jurisprudencia GATT/OMC derivan los siguientes criterios al respecto: características intrínsecas del producto, uso final para el cual se destina el producto, preferencias y comportamiento de los consumidores, clasificación según Sistema Armonizado (SA). origen nacional o de un país tercero. Por tanto, el fin último del principio es evitar que se apliquen requisitos de manera asimétrica entre la producción nacional y las importaciones, que afecten negativamente el acceso de estas al mercado.

\section{b) Prevención de obstáculos innecesarios al comercio}

El Acuerdo OTC establece que al aplicar las medidas sus miembros no tendrán como finalidad generar obstáculos innecesarios al comercio. En este sentido, el concepto de «necesidad» se mediría considerando los riesgos que conllevaría no perseguir los objetivos legítimos que define el propio Acuerdo: imperativos de la seguridad nacional, prevención de prácticas engañosas, protección de la salud o seguridad humanas, protección de la vida o la salud animal o vegetal y protección del medio ambiente. En la evaluación del riesgo debe tenerse presente la información científica y técnica disponible, la tecnología de elaboración conexa y los usos finales a que se destinen los productos.

\section{c) Armonización}

En el actual Acuerdo OTC el contenido del principio de armonización es muy similar al del Acuerdo de 1979. Se establece que los países deben dar prioridad a la utilización (total o parcial) de normas internacionales, si estas existen o están próximas a formularse, como base de sus propios reglamentos técni- 
Sofía Boza y Felipe Fernández • Chile frente a la regulación de las medidas...

cos y normas nacionales. No obstante, el propio Acuerdo abre la posibilidad de no contemplar lo anterior cuando las normas internacionales no sean apropiadas o eficaces para el logro de los objetivos perseguidos. Al respecto, entre las causas de divergencia se mencionan factores climáticos, geográficos o tecnológicos de carácter fundamental.

Para potenciar la viabilidad y cumplimiento de lo señalado el texto del Acuerdo establece que, dentro de sus posibilidades, los países deberán participar «plenamente» en los procesos de elaboración de normas por las «instituciones internacionales competentes con actividades de normalización». A diferencia de lo que sucede en el Acuerdo MSF, el Acuerdo OTC no establece de manera explícita una relación de dichas instituciones internacionales y solo expresa que deben ser abiertas a la participación de todos los miembros. Dada esta falta de precisión, el Comité sobre Obstáculos Técnicos al Comercio ha acordado que, además de la apertu$\mathrm{ra}$, las instituciones internacionales de referencia deben cumplir con los principios de transparencia, imparcialidad, consenso, eficacia, pertinencia, coherencia y consideración del desarrollo ${ }^{2}$.

\footnotetext{
A nivel práctico, algunos ejemplos de «instituciones internacionales competentes con actividades de normalización» en el marco del Acuerdo OTC son: la International Organization for Standardization (ISO), la International Telecommunication Union (ITU) y la Comisión del Codex Alimentarius.
}

\section{d) Equivalencia y reconocimiento mutuo}

De manera complementaria a lo definido bajo el principio de armonización, el Acuerdo alienta a los países miembros a reconocer como equivalentes los reglamentos técnicos de otros países miembros. Lo mismo se establece para los resultados de los procesos de evaluación de conformidad. Para facilitar lo anterior el Acuerdo señala que los reglamentos técnicos sobre prescripciones en productos deben diseñarse sobre la base de «las propiedades de uso y empleo de los productos más bien que en función de su diseño o de sus características descriptivas» (artículo, 2.8). Con esto último se trata de evitar que la justificación para desestimar la equivalencia se fundamente en detalles de forma relacionados con los productos normados y no así en las características que verdaderamente pueden entrar en conflicto con los objetivos legítimos fijados en el Acuerdo.

\section{e) Transparencia}

En materia de transparencia, según el Acuerdo OTC los países miembros se comprometen a: i) anunciar de manera pública su intención de introducir una medida, ii) notificar por conducto de la Secretaría de la OMC los contenidos de dicha medida, iii) facilitar los detalles del proyecto de la medida bajo solicitud y iv) dar tiempo para recibir observaciones de los demás miembros, conversar sobre ellas en caso necesario y conside- 
rar este proceso en la propuesta final. El propio Acuerdo fija excepciones a lo anterior cuando el proceso antes señalado pueda ser contraproducente debido a problemas urgentes relacionados con sanidad, protección del medio ambiente o seguridad nacional. No obstante, en estos casos también deberá realizarse una notificación de la medida a través de la Secretaría de la OMC, identificando los problemas urgentes que lo justifican. Además, se debe facilitar el texto completo de la medida objeto de la solicitud y permitir que los demás países miembros formulen observaciones y conversen al respecto, teniendo en cuenta los resultados de ambos procesos.

\section{f) Asistencia técnica y trato especial y diferenciado}

Este principio es una disposición en materia de trato especial y diferenciado contemplada en diversos acuerdos de la OMC como "concesiones especiales» para los países miembros en desarrollo. En el caso específico del Acuerdo OTC se refleja, por una parte, en la obligación de proveer a los países miembros en desarrollo que así lo soliciten asistencia técnica relacionada con el cumplimiento de los requerimientos, con la generación de instituciones nacionales y con la participación de las instituciones internacionales de referencia. Del mismo modo, el Acuerdo establece que en lo explicitado deben tenerse presentes las particularidades de los países miembros en desarrollo. Esto se traduciría incluso en que, cuando lo estime oportuno, el Comité OTC pueda conceder excepciones al cumplimiento de obligaciones, pero siempre por un periodo limitado y previa solicitud del país interesado.

Además de las disposiciones basadas en los principios señalados, el Acuerdo OTC contempla la creación del Comité OTC, compuesto por representantes de cada uno de los países miembros. El Comité deberá reunirse al menos una vez al año, permitiendo así que los miembros puedan, entre otras cosas, plantear sus inquietudes sobre los requisitos impuestos por otros países miembros. Estos planteamientos se conocen como Preocupaciones Comerciales Específicas (PCE). En este sentido, según señalan Horn, Mavroidis y Wijkström (2013) pueden distinguirse dos categorías, como tales que denominan «serias» 0 «triviales», según la naturaleza del asunto tratado y, de manera más evidente, de acuerdo con el número de reuniones que se requieren para que la PCE salga de la agenda.

No obstante, incluso cuando se planteen Preocupaciones Comerciales Específicas «serias», el Comité OTC carece de atribuciones para pronunciarse formalmente sobre la materia de que tratan. En esas circunstancias, cuando un país miembro tiene la convicción de que otro no está respetando lo establecido en el Acuerdo OTC y con ello perjudica sus posibilidades comerciales, puede plantear el conflicto ante el Órgano de Solución de Diferencias (OSD) de la OMC para 
Sofía Boza y Felipe Fernández • Chile frente a la regulación de las medidas...

someterlo a su dictamen. Dada la especificidad de las materias tratadas, el Acuerdo OTC facilita que el grupo especial respectivo a la disputa, se apoye en la asesoría de un grupo de expertos técnicos ad hoc.

\section{LA PARTICIPACIÓN DE CHILE EN LOS MECANISMOS CON ARREgLO Al ACUERdo OTC}

Al igual que el resto de países miembros de la OMC que suscribieron el Acuerdo OTC, los reglamentos técnicos, normas y procedimientos de evaluación de conformidad que Chile impone quedan sujetos a él, lo que de acuerdo con el principio de transparencia, deriva en la obligación de las autoridades competentes nacionales de notificar la intención de hacer entrar en vigor estas medidas o introducir cambios sustantivos en las existentes. Asimismo, una representación del país debe participar en el Comité OTC, lo que le posibilita plantear Preocupaciones Comerciales Específicas, ser receptor de ellas o apoyar las planteadas por otros miembros. Asimismo, cuando lo considere necesario, Chile puede acceder al Órgano de Solución de Diferencias para tratar de resolver una controversia con otro país miembro en materias relacionadas con el Acuerdo. No obstante, el país también puede ser demandado o participar como tercero.

Considerando los mecanismos institucionales mencionados, a continuación se expondrá cuál ha sido la participación de Chile en cada uno de ellos durante el periodo 1995-2012.

\section{a) Notificaciones}

Según registra la base de datos I-TIP de la OMC, entre 1995 y 2012 se informaron más de quince mil notificaciones sobre OTC a la Secretaría. Dentro de la cifra anterior, los países con mayor número de notificaciones fueron, en el mismo orden, Estados Unidos, China, Israel, Unión Europea, Japón y Brasil. El importante posicionamiento de Brasil como líder en notificaciones de OTC a nivel regional coincide con lo sucedido con las medidas sanitarias y fitosanitarias, en que el país es segundo en el mundo (solo por detrás de Estados Unidos). Según De Almeida, Monteiro da Silva y de Lima (2010) el rápido crecimiento de la economía brasileña, que derivaría en un mayor nivel de exigencia por parte de consumidores y productores en lo relacionado con los aspectos técnicos de las mercancías, es sugerido por De Almeida, Monteiro da Silva \& De Lima (2010) explicaría el elevado número de notificaciones, al menos en MSF.

Para el caso específico de Chile, el país es el decimoquinto a nivel mundial en número de notificaciones en OTC presentadas a la Secretaría de la OMC, con un total de 289 entre 1995 y 2012. En dicho periodo, fueron especialmente prolíficos los años 1999 a 2001, así como 2010 a 2012. Parte importante de las notificaciones se han relacionado con reglamentos 
técnicos, normas y procedimientos de evaluación de conformidad sobre productos agroalimentarios. Dentro de lo anterior, la tipificación de la carne, los insumos de la cría animal, la regulación sobre agricultura orgánica, la calidad y variedad de las semillas, las normas sobre insumos agrícolas y lo referente a la producción vinícola, han sido los aspectos específicos de mayor interés en los OTC chilenos.

A nivel operativo, en Chile la administración del Acuerdo OTC corresponde al Ministerio de Relaciones Exteriores, el que delega en la Dirección General de Relaciones Económicas Internacionales (DIRECON) la función de punto de contacto con la Secretaría de la OMC para informar sobre las notificaciones sobre OTC. En este contexto, el Reglamento de Ejecución para la Elaboración y Adopción de Reglamentos Técnicos, promulgado en Chile en 2004 constituye una guía esencial de buenas prácticas regulatorias en OTC para los distintos organismos del país competentes en la materia, que se refiere asimismo a los procedimientos para la notificación. Este Reglamento, surgido al amparo de la Ley 19.912 de 2003 de adecuación a la OMC, resulta de especial interés, pues introduce principios que antes no estaban explicitados en la legislación chilena (Rojas Vega, 2012). De hecho, el Decreto establece que los reglamentos técnicos y procedimientos de evaluación de conformidad se dictarán atendiendo a prácticas que persiguen la consecución de los principios de armonización, prevención de obstáculos innecesarios al comercio, no discriminación, equivalencia y reconocimiento mutuo.

\section{b) Preocupaciones comerciales especificas}

En lo que se refiere a las preocupaciones comerciales específicas (PCE) presentadas al Comité OTC, según datos de la OMC entre 1995 y 2012 alcanzaron un total de 363 , centradas principalmente en el periodo 20052009. La Unión Europea y Estados Unidos son los miembros que han tenido mayor participación en el planteamiento de estas Preocupaciones, con 177 y 149 respectivamente. Por otra parte, en lo que se refiere al país que mantiene la medida por la cual se elevó la PCE, los miembros emplazados en más ocasiones han sido la Unión Europea (80 PCE), China (42 PCE) y Estados Unidos (41 PCE). En este contexto, destaca también la participación muy activa en el planteamiento de Preocupaciones Comerciales Específicas de algunos países de América Latina como México (47 PCE), Argentina (29 PCE) y Brasil (25 PCE). Al grupo señalado se podría agregar el caso de Chile, ya que el país presentó veintiuna PCE en el periodo en estudio, mientras que sus medidas fueron cuestionadas solamente en dos ocasiones. 
Sofía Boza y Felipe Fernández • Chile frente a la regulación de las medidas...

Cuadro 2. PCE con Chile presentadas por los países al Comité OTC

\begin{tabular}{|c|c|c|c|}
\hline $\begin{array}{l}\text { Miembro } \\
\text { que } \\
\text { mantiene } \\
\text { OTC }\end{array}$ & $\begin{array}{l}\text { Primera } \\
\text { presen- } \\
\text { tación }\end{array}$ & $\begin{array}{l}\text { Última } \\
\text { presentación }\end{array}$ & Tema(s) de la medida \\
\hline $\begin{array}{l}\text { Unión } \\
\text { Europea }\end{array}$ & $\begin{array}{l}\text { Oct.- } \\
1999\end{array}$ & Nov.-2012 & $\begin{array}{l}\text { Regulación sobre ciertos productos } \\
\text { vinícolas }\end{array}$ \\
\hline Ecuador & $\begin{array}{l}\text { Julio } \\
2003\end{array}$ & Marzo-2004 & $\begin{array}{l}\text { Normas sobre barras de acero laminadas } \\
\text { en caliente para hormigón armado }\end{array}$ \\
\hline $\begin{array}{l}\text { Unión } \\
\text { Europea }\end{array}$ & $\begin{array}{l}\text { Julio } \\
2003\end{array}$ & Julio 2003 & $\begin{array}{l}\text { Modificaciones a la regulación en } \\
\text { sardinas }\end{array}$ \\
\hline $\begin{array}{l}\text { Unión } \\
\text { Europea }\end{array}$ & $\begin{array}{l}\text { Nov. } \\
2005\end{array}$ & Marzo 2013 & $\begin{array}{l}\text { Regulación en registro, evaluación y } \\
\text { autorización de químicos }\end{array}$ \\
\hline $\begin{array}{l}\text { Estados } \\
\text { Unidos }\end{array}$ & $\begin{array}{l}\text { Marzo } \\
2008\end{array}$ & Marzo 2010 & Regulación química antiterrorista \\
\hline Colombia & $\begin{array}{l}\text { Julio } \\
2008\end{array}$ & Julio 2008 & Regulación sobre etiquetado de vino \\
\hline $\begin{array}{l}\text { Unión } \\
\text { Europea }\end{array}$ & $\begin{array}{l}\text { Julio } \\
2008\end{array}$ & Julio 2008 & $\begin{array}{l}\text { Cambios en Alemania a la ordenanza } \\
\text { sobre prohibición de químicos }\end{array}$ \\
\hline Argentina & $\begin{array}{l}\text { Marzo } \\
2009\end{array}$ & Junio 2009 & $\begin{array}{l}\text { Medidas que afectan el acceso al } \\
\text { mercado de productos farmacéuticos }\end{array}$ \\
\hline Ecuador & $\begin{array}{l}\text { Marzo } \\
2009\end{array}$ & Marzo 2009 & $\begin{array}{l}\text { Informe de prueba y certificado de } \\
\text { conformidad para productos de la } \\
\text { industria automotriz }\end{array}$ \\
\hline $\begin{array}{l}\text { Rep. de } \\
\text { Corea }\end{array}$ & $\begin{array}{l}\text { Junio } \\
2009\end{array}$ & Nov. 2010 & $\begin{array}{l}\text { Regulación sobre promoción } \\
\text { agroalimentaria }\end{array}$ \\
\hline Canadá & $\begin{array}{l}\text { Nov. } \\
2009\end{array}$ & Marzo 2011 & Modificaciones a la ley de tabaco \\
\hline $\begin{array}{l}\text { Unión } \\
\text { Europea }\end{array}$ & $\begin{array}{l}\text { Nov. } \\
2009\end{array}$ & Junio 2011 & $\begin{array}{l}\text { Regulación en clasificación, etiquetado } \\
\text { y empaquetamiento de sustancias y } \\
\text { mixturas }\end{array}$ \\
\hline Tailandia & $\begin{array}{l}\text { Marzo } \\
2010\end{array}$ & Marzo 2012 & $\begin{array}{l}\text { Advertencias sanitarias para bebidas } \\
\text { alcohólicas }\end{array}$ \\
\hline Viet Nam & $\begin{array}{l}\text { Junio } \\
2010\end{array}$ & Nov. 2010 & Bebidas alcohólicas \\
\hline Brasil & $\begin{array}{l}\text { Marzo } \\
2011\end{array}$ & Junio 2012 & $\begin{array}{l}\text { Máximos niveles permitidos de alquitrán, } \\
\text { nicotina, monóxido de carbono y } \\
\text { aditivos en tabaco }\end{array}$ \\
\hline
\end{tabular}




\begin{tabular}{|l|l|l|l|}
\hline Australia & $\begin{array}{l}\text { Junio } \\
2011\end{array}$ & Junio 2012 & $\begin{array}{l}\text { Regulación sobre empaquetamiento del } \\
\text { tabaco }\end{array}$ \\
\hline Viet Nam & $\begin{array}{l}\text { Junio } \\
2011\end{array}$ & Junio 2012 & $\begin{array}{l}\text { Procedimientos de conformidad en } \\
\text { alcohol, cosméticos y teléfonos celulares }\end{array}$ \\
\hline Perú & $\begin{array}{l}\text { Nov. } \\
2011\end{array}$ & Marzo 2013 & Regulación en etiquetado de transgénicos \\
\hline $\begin{array}{l}\text { Estados } \\
\text { Unidos }\end{array}$ & $\begin{array}{l}\text { Junio } \\
2012\end{array}$ & Junio 2012 & $\begin{array}{l}\text { Normas sobre identificación de pisco y } \\
\text { coñac }\end{array}$ \\
\hline Viet Nam & $\begin{array}{l}\text { Junio } \\
2012\end{array}$ & Nov. 2012 & $\begin{array}{l}\text { Regulación en producción y comercio de } \\
\text { licor }\end{array}$ \\
\hline Viet Nam & $\begin{array}{l}\text { Junio } \\
2012\end{array}$ & Marzo 2013 & Legislación sobre inocuidad alimentaria \\
\hline
\end{tabular}

Como se puede observar en el Cuadro 2, los principales miembros que mantuvieron las medidas frente a las que Chile se pronunció durante el periodo en estudio a partir del planteamiento de una PCE son otros países de América Latina (Argentina, Brasil, Colombia, Ecuador y Perú), la Unión Europea y algunas naciones del sudeste asiático (Tailandia y Viet Nam). Por su parte, los productos más citados en estas medidas son bebidas alcohólicas, especialmente el vino, y tabaco.

\section{c) Diferencias}

En investigaciones recientes diversos autores han identificado a nivel general (no solo para OTC) las condiciones que consideran relacionadas con el nivel de actividad de los países en el OSD. Algunas de ellas, como poder económico (Sattler \& Bernauer, 2011), capacidad legal (Busch, Reinhardt \& Shaffer, 2008; Conti, 2010), diversidad y valor de las exportaciones (Horn, Mavroidis
\& Wijkström, 1999; Holmes, Rollo \& Young, 2003) y disponibilidad de recursos financieros, humanos e institucionales (Bohl, 2009; Guzmán \& Simmons, 2005), sugieren una relación entre el nivel de desarrollo del país y la frecuencia con que este recurre al OSD. De hecho, Leitner \& Leister (2013) después de analizar las principales tendencias en el total de disputas ante la OMC entre 1995 y 2012 que Estados Unidos y la Unión Europea serían por lejos los miembros con mayor participación como reclamantes, así como demandados, ante el OSD.

Para el caso específico de las controversias planteadas ante la OMC en que se citó el Acuerdo OTC, se cumple también lo señalado en el párrafo anterior, ya que en los 45 casos iniciados entre 1995 y 2012, los países con mayor participación como reclamantes fueron Canadá (10 casos), Estados Unidos (9 casos) y la Unión Europea (5 casos). De manera similar, la Unión Europea y Estados Unidos fueron los países 
Sofía Boza y Felipe Fernández • Chile frente a la regulación de las medidas...

demandados en más ocasiones, con 19 cierta participación en las disputas bajo y 11 casos respectivamente. No obs- el Acuerdo OTC. Es el caso de Chile, tante, a pesar de la preponderancia de que ha actuado como demandante en los países más desarrollados, algunos dos diferencias y como tercero en cinco miembros de ingreso medio han tenido de ellas (Cuadro 3).

Cuadro 3. Participación de Chile en disputas con arreglo al Acuerdo OTC

\begin{tabular}{|l|l|l|l|l|l|}
\hline Código & Año & $\begin{array}{l}\text { Recla- } \\
\text { mante }\end{array}$ & $\begin{array}{l}\text { Deman- } \\
\text { dado }\end{array}$ & $\begin{array}{l}\text { Tercera parte } \\
\text { Droducto(s) } \\
\text { Asunto(s) }\end{array}$ \\
\hline DS14 & 1995 & Canadá & CE & $\begin{array}{l}\text { Australia, Chile, Islandia, } \\
\text { Japón, Perú, EEUU }\end{array}$ & Moluscos \\
\hline DS231 & 2001 & Perú & CE & $\begin{array}{l}\text { Australia, Canadá, } \\
\text { Islandia, Japón, EEUU }\end{array}$ & Moluscos \\
\hline DS232 & 2001 & Chile & México & $\begin{array}{l}\text { Ninguna Chile, Colombia, } \\
\text { Ecuador, Venezuela, EEUU }\end{array}$ & Sardinas \\
\hline DS291 & 2003 & EEUU & CE & $\begin{array}{l}\text { Argentina, Australia, } \\
\text { Brasil, Canadá, Chile, } \\
\text { China, Taiwán, Colombia, } \\
\text { El Salvador, Honduras, } \\
\text { México, N. Zelanda, } \\
\text { Noruega, Paraguay, Perú, } \\
\text { Tailandia, Uruguay }\end{array}$ & Biotecnología \\
\hline DS292 & 2003 & Canadá & CE & $\begin{array}{l}\text { Argentina, Australia, } \\
\text { Brasil, Chile, China, } \\
\text { EEUU, Taiwán, Colombia, } \\
\text { El Salvador, Honduras, } \\
\text { México, N. Zelanda, } \\
\text { Noruega, Paraguay, Perú, } \\
\text { Tailandia, Uruguay }\end{array}$ & Biotecnología \\
\hline DS293 & 2003 & Argentina & CE & $\begin{array}{l}\text { Australia, Brasil, Canadá, } \\
\text { Chile, China, EEUU, } \\
\text { Taiwán, Colombia, El } \\
\text { Salvador, Honduras, } \\
\text { México, N. Zelanda, } \\
\text { Noruega, Paraguay, Perú, } \\
\text { Tailandia, Uruguay }\end{array}$ & Biotecnología \\
\hline
\end{tabular}


A continuación se detallan los dos casos en que Chile actuó como demandante ante el OSD en materias regidas por el Acuerdo OTC.

Denominación comercial de los moluscos del género pectinidae (DS12 y DS14)

El sector pesquero es una de las actividades con mayor desarrollo en Chile en los últimos años, destacando dentro del mismo la alta rentabilidad de la producción y comercialización de moluscos bivalvos. De hecho, Chile se ubica entre los tres primeros productores mundiales de mitílidos y pectínidos de cultivo, siendo de especial relevancia el chorito (Mytilus chilensis) y el ostión del norte (Argopecten purpuratus Lamarck), este último muy enfocado al comercio exterior. El principal destino de exportación de estos productos (frescos, en conserva, en semi conserva y congelados) es la Unión Europea, principalmente España, Portugal y Francia (Cantillánez, Thouzeau \& Avendaño, 2007; Lovatelli, Farias \& Uriarte, 2008; Sernapesca, 2012).

Precisamente en Francia, principal consumidor mundial de pectínidos, estos han recibido tradicionalmente la denominación comercial de "coquilles Saint-Jacques» o "noix de coquille Saint-Jacques», tanto para los especímenes de origen francés como para los importados. No obstante, en 1993 el gobierno francés por medio de su autoridad alimentaria, cambió de criterio indicando mediante el Decreto $n^{\circ}$ NOR MERP9300051 A que las denominaciones comerciales «coquille Saint-Jacques» $\mathrm{y}$ «noix de coquille Saint-Jacques» para los moluscos del género de los pectínidos serían en adelante de exclusiva para los producidos en Francia, dejando la denominación "petoncle» para los provenientes de otros países, con el objeto de mejorar la calidad de la información en manos de los consumidores.

En 1995 el gobierno de Perú solicitó a la Comunidad Europea (CE) la celebración de consultas respecto de la medida adoptada por Francia, fundamentándose en el párrafo primero del artículo XXII del GATT 1994, el artículo 14.1 del Acuerdo sobre Obstáculos Técnicos al Comercio y el artículo 4 del Entendimiento relativo a las normas y procedimientos por los que se rige la solución de diferencias. En el mismo año, los gobiernos de Chile y Canadá pidieron sumarse a las consultas solicitadas por Perú.

Dado que las consultas realizadas no permitieron solucionar la diferencia, Perú pidió al Órgano de Solución de Diferencias que estableciera un grupo especial para que este revisara la compatibilidad de la normativa francesa y los artículos antes señalados, medida seguida por Chile y a la que solicitaron asociarse Canadá y Japón. La acción de dicho grupo especial no tampoco permitió alcanzar una solución, por lo que posteriormente, ese mismo año, Chile solicitó al OSD que estableciera otro grupo especial que estudiara si las modificaciones impuestas por Francia eran incompatibles con los artículos I y III del GATT de 1994, y los artículos 2 y 12 del Acuerdo OTC, señalando que 
Sofía Boza y Felipe Fernández • Chile frente a la regulación de las medidas...

se estaba causando menoscabo y anulación a Chile. Conforme a la solicitud antes mencionada, en la siguiente reunión celebrada por el OSD, este resolvió que se constituyera un grupo especial que examinara el asunto y cuyo apoyo le permitiera (al OSD) resolver la diferencia. En dicha reunión, Australia, Canadá, Islandia, Japón y Estados Unidos se reservaron el derecho de intervenir como terceros en el procedimiento del grupo especial.

Después de que el grupo especial diera a conocer a las partes su informe preliminar en febrero de 1996, estas solicitaron un aplazamiento para la entrega de un documento definitivo, dado que se encontraban negociando los términos de una solución mutuamente convenida. De hecho, en julio del mismo año las delegaciones notificaron al OSD que habían logrado la solución, que fue informada a la OMC. En ella se contemplaba que en adelante los moluscos de la familia de los pectínidos debieran comercializarse con la denominación «Saint-Jacques», acompañada del nombre científico de la especie y de la indicación del país de origen; todo ello visible en la misma cara de la etiqueta.

Medidas que afectan a las importaciones de fósforos (DS232)

La República de México cuenta con una serie de instrumentos jurídicos y normas para la elaboración, comercialización, transporte y medición de productos químicos y explosivos, como la Ley de Control de Armas y
Explosivos, la Ley Federal sobre Metrología y Normalización y su respectivo Reglamento y el Reglamento para el Transporte Terrestre de Materiales y Residuos Peligrosos.

En este contexto, en el año 1993 el Gobierno de México a través de la Secretaría de Defensa Nacional dictó una medida que atribuía a los fósforos de seguridad (en México cerillos) provenientes de Chile la categoría de producto explosivo riesgoso, lo que obligaba a que el producto cumpliera con una serie de requisitos extraordinarios relacionados con el embalaje, internación, transporte y almacenamiento. La medida se justificó aduciendo que la denominación chilena de «fósforo» o «fósforo de seguridad» creaba confusión con el elemento químico homónimo.

Como respuesta a dicha medida, el 17 de mayo de 2001 Chile solicitó a México y al Órgano de Solución de Diferencias la celebración de consultas, dado que a su juicio estas medidas constituían obstáculos innecesarios al acceso al mercado mexicano para los fósforos de seguridad provenientes del país. La solicitud presentada por Chile señala que las medidas impuestas por México eran incompatibles con los artículos 1, 2 y 5 del Acuerdo de Obstáculos Técnicos al Comercio de la OMC, con los artículos 1,3 y 5 del Acuerdo sobre Procedimientos para el Trámite de Licencias de Importación y con el párrafo 4 del artículo III del GATT de 1994. En el mes de junio del 2001, la Comisión Europea solicitó asociarse 
a las consultas solicitadas por Chile, debido a su interés comercial.

Posteriormente, los gobiernos de Chile y México mantuvieron conversaciones periódicas con el fin de buscar una solución convenida a la diferencia planteada. Efectivamente, el 2 de febrero de 2004, Chile emitió un comunicado al Presidente del Órgano de Solución de Diferencias, señalando que, producto de las constructivas gestiones realizadas entre los dos países en el conflicto, retiraba la solicitud de la consulta por este caso, poniéndole término.

\section{Conclusiones}

La entrada en vigor del Acuerdo OTC en 1995 constituyó un marco para que los países miembros establecieran medidas técnicas. $\mathrm{Al}$ respecto, uno de los principales logros del Acuerdo fue definir una serie de principios para evitar que las medidas sean utilizadas con fines relacionaos con el deseo de proteger los mercados internos de la competencia internacional. Por una parte, dada la amplitud de criterios en que se basan los OTC y el importante componente técnico de estos, es muy importante proteger la transparencia, de manera que todos los países miembros puedan contar con información completa sobre las medidas y formular sus comentarios. Por otra parte, las disposiciones relacionadas con los principios de armonización y equivalencia procuran limitar la profusión de reglamentos, normas y procedimientos de evaluación de conformidad.

De igual modo, el Acuerdo OTC crea como hemos visto un Comité ad hoc, compuesto por todos los países miembros, que se reúne periódicamente y en el que se exponen las preocupaciones existentes frente a las medidas tomadas por otros países. En el mismo sentido, el Acuerdo fija algunas pautas particulares para el funcionamiento de la solución de diferencias ante la OMC en lo que respecta a OTC. Todo ello constituye un importante marco institucional para potenciar la generación de consenso y la solución de conflictos.

El grado de participación que cada país ha mostrado en los mecanismos señalados, además de en la generación de notificaciones, ha sido muy dispar. $A$ priori, queda de manifiesto que los países más desarrollados, principalmente Estados Unidos y la Unión Europea, han tenido una participación preeminente en los mecanismos del Acuerdo OTC. Sin embargo, algunos países de renta media también han participado activamente como miembros del Acuerdo. Es el caso de Chile, que destaca por el número de notificaciones en OTC, siendo uno de los más prolíficos en América Latina y decimoquinto a nivel mundial. En rigor, el número de notificaciones en OTC del país ha registrado una tendencia al alza muy acentuada desde 2010. Algo similar ha sucedido con el planteamiento de Preocupaciones Comerciales Específicas ante el Comité OTC, donde de las veintiuna presentadas por Chile en el periodo en estudio, 
Sofía Boza y Felipe Fernández • Chile frente a la regulación de las medidas...

diecisiete lo fueron a partir de 2008. En cambio, Chile solo ha participado como demandante en dos oportunidades, llegando en ambos casos a soluciones convenidas por las partes. Sin embargo, en un número mucho más significativo el país ha solicitado intervenir como tercero.

En general, podemos por tanto decir que pese a su pequeño tamaño (en términos de población) y su relativamente menor nivel económico (en términos absolutos), Chile ha sido un país muy activo en la aplicación de medidas técnicas relacionadas con el comercio y en su participación en el Comité OTC. En este sentido, se evidencia una importante capacidad institucional del país, así como su voluntad de prestar atención preferente a lo referido a sus importaciones y exportaciones. Esta inserción resulta de especial interés, en un escenario en el cual los aranceles tienen cada vez menor impacto en las relaciones comerciales entre países (sobre todo para economías tan abiertas como la chilena) mientras que aumenta gradualmente la importancia de las medidas no arancelarias.

\section{Bibliografía}

Banco Mundial. (2008). A Survey of Non-Tariff Measures in the East Asia and Pacific Region. Washington DC: Banco Mundial.

Bohl, Kristin (2009), «Problems of developing country access to the WTO Disputes Settlement», Chicago-Kent Journal of International \& Comparative Law, pp. 130-200.

Bush, Marc L., Eric Reinhardt y Gregory Shaffer (2008), «Does legal capacity matter? Explaining dispute initiation and antidumping actions in the WTO». International Centre for Trade and Sustainable Development, issue paper $n^{\circ} 4$.

Cantillánez, Marcela, Gérard Thouzeau y Miguel Avendaño (2007), «Improving Argopecten purpuratus culture in northern Chile: Results from the study of larval and post-larval stages in relation to environmental forcing», Aquaculture, vol. 272, issues 1-4, pp. 423-443.

Conferencia de las Naciones Unidas para el Comercio y el Desarrollo, (UNCTAD) (2010). Non-tariff measures: Evidence from Selected Developing Countries and Future Research Agenda. Ginebra: Publicaciones de las Naciones Unidas.

Conti, Joseph A. (2010), «Learning to dispute: repeat participation, expertise, and reputation at the World Trade Organization", Law \& Social Inquiry, vol. 35, issue 3, pp. 625-662.

Da Almeida, Fernanda María, Orlando Monteiro da Silva y Joao Eustaquio Da Lima (2010). «Determinantes das notificações aos acordos sobre medidas SPS e TBT emitidas pelo Brasil». En Orlando Monteiro da Silva (ed.): Notificações aos Acordos de Barreiras Técnicas (TBT) e Sanitárias (SPS) da OMC: transparência comercial ou barreiras não tarifárias? (pp. 155-180). Viçosa: Universidade Federal de Viçosa.

Guzmán, Andrew T. y Beth A. Simmons (2005), «Power plays \& capacity constraints: the selection of defendants in WTO Disputes», UC Berkeley Public Law Research Paper $n^{\circ} 660501$.

Hobbs, Jill E. (2010), "Public and Private Standards for Food Safety and Quality: International Trade Implications», Estey Centre Journal of International Law and Trade Policy, vol. 11, n 1, pp. 136-152.

Holmes, Peter, Jim Rollo y Alastair R. Young (2003), «Emerging trends in the WTO Disputes Settlement: Back to the GATT?", World Bank Policy Research Working Paper $n^{\circ} 3133$.

Horn, Henrik, Petros C. Mavroidis y Hakan Nordström (1999), «Is The Use Of The 
WTO Dispute Settlement System Biased?», CEPR Discussion Papers n 2340.

Horn, Henrik, Petros C.Mavroidis y Enrik N.

Wijkström (2013), "In the Shadow of the DSU: Addressing Specific Trade Concerns on the WTO SPS and TBT Committees». Journal of World Trade, vol. 47, issue 4, pp. 729-759.

Leitner, Kara y Simon Lester (2013), «WTO Disputes Settlement 1995-2012: A statistical analysis", Journal of International Economic Law, vol. 16, issue 1, pp. 257-267. Lovatelli, Alessandro, Ana Farias e Iker Uriarte (2008), Estado actual del cultivo y manejo de moluscos bivalvos y su protección futura: Factores que afectan su sustentabilidad en América Latina. Roma: FAO.

Organización de las Naciones Unidas para la Agricultura y la Alimentación FAO (2000), Las negociaciones comerciales multilaterales sobre la agricultura III: Acuerdo sobre la Aplicación de Medidas Sanitarias y Fitosanitarias y Acuerdo sobre Obstáculos Técnicos al Comercio. Roma: FAO.
Organización Mundial del Comercio (OMC) (2012), World Trade Report 2012. Ginebra: OMC.

Rojas Vega, Oscar (2012), Coherencia regulatoria, buenas prácticas para regular. (Tesis Magíster en Estrategia Internacional y Política Comercial) Universidad de Chile. Sattler, Thomas y Thomas Bernauer (2011), «Gravity or discrimination? Determinants of litigation in the World Trade Organization", European Journal of Political Research, vol. 50, issue 2, 143-167.

Servicio Nacional de Pesca (SERNAPESCA) (2012). Exportación de mitílidos a la UE. Recuperado el 19 de diciembre de 2013 de http:// www.sernapesca.cl/index.php?option $=$ com content $\&$ view $=$ category $\&$ layout $=$ blog $\& \mathrm{id}=\overline{2}$ 29\&Itemid $=1018$

Van Tongeren, Frank, John Beghin y Stephan Marette (2009), "A cost-benefit framework for the assessment of non-tariff measures in agro-food trade», OECD Food Agriculture and Fisheries Working Papers $n^{\circ} 21$. 